\title{
POLIQUETOS ERRANTES DE LA PLATAFORMA CONTINENTAL DE TAMAULIPAS, MÉXICO
}

\section{ERRANT POLYCHAETES OF THE CONTINENTAL PLATFORM OF TAMAULIPAS, MEXICO}

\author{
V. Hugo Delgado-Blas *
}

\section{RESUMEN}

Se registran 24 especies de poliquetos errantes para la plataforma continental oriental mexicana: Dos Phyllodocidae, uno Pilargidae, dos Syllidae, cuatro Nereididae, dos Glyceridae, dos Goniadidae, tres Nephtyidae, dos Onuphidae, cinco Lumbrineridae y uno Dorvilleidae. De ellos, dos se registran por primera vez para México, dos para aguas mexicanas del Golfo de México y 11 son nuevos registros para Tamaulipas.

Palabras clave: Poliquetos, ecología, Golfo de México.

\begin{abstract}
24 species of errant polychaetes have been recorded for the eastern Mexican continental shelf: Two Phyllodocidae, one Pilargidae, two Syllidae, lour Nereididae, two Glyceridae, two Goniadidae, three Nephtyidae, two Onuphidae, five Lumbrineridae y one Dorvlleidae. Two of these were the first to be recorded in Mexico, two from Mexican waters in the Gulf of Mexico and 11 new ones recorded in Tamaulipas.
\end{abstract}

Key words: Polychaetes, ecology, Gulf of Mexico.

\section{INTRODUCCIÓN}

El litoral del sur de Tamaulipas es un sistema ecológicamente complíjo e interesante, ya que por su siluación geográfica recibe aporles de masas de agua epicontinentales así como de orjgen tropical, subtropical y templado, además de lagunas costeras; todos estos factores limnológicos y oceanográlicos dan lugar a una gran diversidad de hábitats.

Se ha intensificado el interés por conocer la fauna de la plataforma continental, ya que existen escasos registros de la diversidad bénlica,

Ingenjeria Ambiental, UQROO. Apdo. Postal 10, Chefumal ORoo 77018 México Fax (983) 29056/ e-mali: blas @ balam cuc. uqroo mx especialmente poliquetos. Este taxón es generalmente el más abundante, frecuente y diversificado entre la macrofauna béntica (Fauchald y Jumars 1979; Salazar-Vallejo et al. 1989). La gran diversidad que exhiben en sus modos de vida y el tipo de alimentación juegan un papel importante dentro de sas redes tróficas y comúnmente sirven de alimento a muchas especies marinas de interés comercial (HernándezAlcántara y Solís-Weis‘, 1993). Además se distribuyen en todos los océanos, cstuarios y en algunos ambientes dulceacuícolas (Fauchald, 1972)

Hasta hace poco, e! conocimiento de la fauna de los poliquetos errantes de la plataforma continental de Tamaulipas sólo consistía en da- 
tos parciales, casi todos restringidos a las zonas más estudiadas que son el norte y sur del Golfo de México (Veracruz, Tabasco, Campeche), y se carecía de datos referentes al litoral de Tamaulipas. Los trabajos de Dexter (1976), Harper et al. (1979), Salazar-Vallejo (1981), Méndez-Ubach (1983), De León-González (1985), Miranda-Vázquez (1993), RodríguezVillanueva (1993) y Solís-Weiss et al. (1995) suplen la falta anterior de conocimiento de los poliquetos en la plataforma oriental del Golfo de México. Cabe destacar que todos estos estudios fueron desarrollados en grandes extensiones geográficas con una o dos estaciones en el litoral de Tamaulipas, y la mayoría son de la zona intermareal.

Como se observa, no existía hasta la fecha un trabajo formal donde se analizara únicamente las poblaciones de poliquetos bénticos de la plataforma continental de Tamaulipas, pues los estudios existentes son de una amplia distribución geográfica; por ello la presente investigación tiene el objetivo de dar a conocer la composición específica y distribución de los anélidos poliquetos errantes de Tamaulipas.

\section{ÁREA DE ESTUDIO}

El área de estudio se localiza en la plataforma continental de Tamaulipas y norte de Veracruz $\left(25^{\circ} 50^{\prime}-20^{\circ} 50^{\prime} \mathrm{N}\right.$ y $97^{\circ} 0^{\prime}-96^{\circ} 50^{\prime} \mathrm{W}$ ) (Fig. 1). La plataforma oriental mexicana se extiende a partir del delta del río Bravo hasta el norte de Veracruz con una amplitud de 33 - 80 km (Lugo 1985). Las corrientes están relacionadas con la influencia de las aguas cálidas y salinas del Caribe que entran a través del estrecho de Yucatán y salen por Florida. La salinidad superficial es mayor de $36 \%$ aunque varía de 34,4 a $36,5 \%$ y la temperatura superficial es de 25,5 a $29^{\circ} \mathrm{C}$ (Vidal et al. 1994). Los sedimentos están dominados por lodos terrígenos, debido a los ríos que desembocan en esta zona y contribuyen a la formación de una franja de limos arenosos terrígenos (Pica et al. 1991).

\section{MATERIALES Y MÉTODO}

El muestreo fue realizado a bordo del $\mathrm{B} / \mathrm{O}$ Antares H-06 en mayo de 1994; con una draga Smith-McIntyre de $\left(0,1 \mathrm{~m}^{2}\right)$ a profundidades de 10 a $130 \mathrm{~m}$, en 15 estaciones. Una vez extraído el sedimento se lavó a través de tamices de 0,5 y 1,0 mm de luz de malla; el material biológico retenido en los tamices se fijó con formol comercial al 10\%; posteriormente en el laboratorio se lavaron nuevamente las muestras con agua corriente para eliminar el formol; el material se separó con un microscopio estereoscópico a nivel de taxa y se preservó en etanol al 70\%, y se rotuló para su posterior análisis.

En la identificación de los poliquetos se siguió la metodología estándar, utilizando las claves taxonómicas de Hartman (1963), Day (1967), Fauchald (1977), Uebelacker \& Johnson (1984a), Salazar-Vallejo et al. (1989)

La lista sistemática sigue el orden filogenético de Fauchald (1977) para cada una de las especies, se citan referencias que ayudaron a su determinación específica y su distribución geográfica; se presentan los datos de estación y entre paréntesis se incluye el número de organismos de cada especie; el hábitat en el área de estudio y la distribución mundial y en México.

\section{RESULTADOS}

Se identificaron 24 especies de poliquetos errantes de 10 familias, de las cuales dos se registran por primera vez para México; dos para aguas mexicanas del Golfo de México y 11 son nuevos registros para esta región.

FAMILIA PHYLLODOCIDAE Williams, 1851

Eteone heteropoda Hartman, 1951

Referencias. Hartman 1951; 31, Lám. 9, figs. 1-8; Pettibone 1963: 72, fig. 16d; Day 1973: 19; Gathof 1984: 19.6, fig. 19.2a-g; Salazar-Vallejo 1996: 115, fig. 13.

Material examinado. 2 organismos: 6(1), 12(1). 


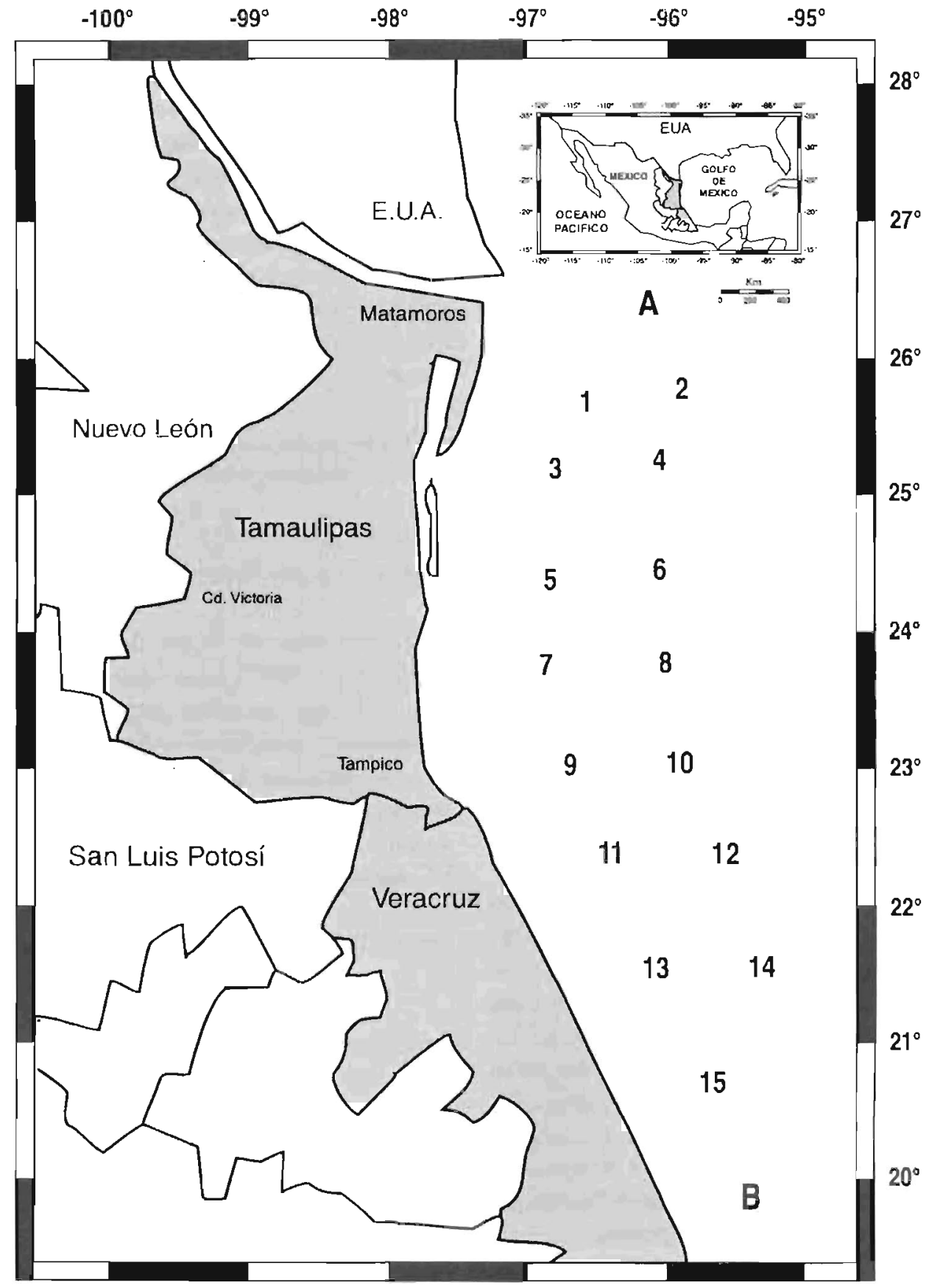

Figura 1. Litoral de Tamaulipas y Norte de Veracruz. A. Ubicación geográfica, B. Estaciones de muestreo 
Distribución. Atlántico occidental. Se encuentra ampliamente distribuida desde el Atlántico noroccidental hasta el Caribe y en el norte del Golfo México hasta Tampico, Tamaulipas y Campeche.

Hábitat. Desde la zona intermareal hasta 200 $m$ de profundidad, se ha encontrado en fondos lodosos y en conchilla; en este trabajo se encontró en sedimento limoso a 56-81 m.

Paranaitis speciosa (Webster, 1880)

Referencias. Pettibone 1903: 75, fig. 17a; Day 1973:22; Gathof 1984: 19-23, fig. 19-19, 20a-e; Rodríguez Villanueva 1993.

Material examinado. 1 organismo: 14(1).

Distribución. Atlántico occidental, desde Maine hasta Alabama, norte del Golfo de México. En aguas mexicanas se ha registradoen el litoral de Campeche; ésta es la primera cita para Tamaulipas.

Hábitat. Desde la zona intermaleal hasta 185 $m$ de profundidad en arcillas hasta fragmentos de conchas; epibionte de Mytilus edulis y sobre tubos de Diopatra cuprea. Ein este trabajo se halló en sedimento limoso arenoso a $60 \mathrm{~m}$.

FAMLIA PILARGIDAE Saint-Joseph, 1899 Sigambra tentaculata (Treadwell, 1941)

Referencias. Pettibone 1966: 182, figs. 14-15; Hartman 1968: 391, figs. 1-3; Gardiner 1976: 121, fig. 9c; Wolf 1984: 29.8, figs. 29.6a-h; HemándezAlcántara 1992: 192; Granados Barba 1994: 138 , Lám. 20a,g.

Material examinado. 1 organismo 12(1).

Distribución. Cosmopolita. Se ha registradoen el mar Negro, mar Rojo y sur de África, en México se ha citado para el Golfo de California, Jalisco, Sinaloa, Nayarit, y se encuentra ampliamente distribuido en el Golfo de México.

Hábitat. Desde la zona intermareal hasta 5121 $m$ de profundidad; se ha encontrado en una amplia variedad de sedimentos desde lodo hasta fragmentos do conchas. En este trabajo se encontró en limos a $81 \mathrm{~m}$

FAMILIA SYLLIDAE Grube, 1850 Spherosyllis glandulata Perkins, $198 \mathrm{I}$

Referencias. Pcrkins 1981: 1123, figs. 18a-c, 19a-j. Uebelacker \& Jonson 1984b: 30-33, figs. 30-25, 26a-e.

Material examinado. 4 organismos: 1(4).

Distribución. Atlántico occidental. Carolina de! Norte, Florida, norte del Golfo de México. Este es el primer registro para aguas mexicanas.

Hábitat. Se ha encontrado en fondo arenoso de arena fina; se encontró en arena limosa a $27 \mathrm{~m}$.

Spherosyllis taylori Perkins, 198 ?

Referencias. Perkins 1981: I 140, fig. 26a-k; Uebelacker \& Johnson 1984b: 30-29, fig. 30.21, 22a-f; Miranda Vázquez 1993.

Material examinado. 2 organismos: 8(2).

Distribución. Atlántico occidental. Connecticut, Maryland, Florida, norte del Golfo de México, en aguas mexicanas se ha registrado para Campeche y ésta es la primera cita para Tamaulipas.

Hábitat. Desde la zona intermareal hasta $90 \mathrm{~m}$ en arena fina a gruesa; en este estudio se encontró a $52 \mathrm{~m}$ en arena

FAMILIA NEREIDIDAE Johnston, 1845 Neanthes succinea (Frey \& Leuckart, 1847)

Referencias. Pettibone 1963:165, figs. 44a-e, 45a-d; Day 1973: 41; Gardiner 1976: 149, figs. 14p, 15a-d; Granados Barba 1994; Taylor 1984 $31-17$, fig. 31.15, 16a-h

Material examinado. 6 organismos: I (3), 3(1). Distribución. Cosmopolita, aguas templadas y tropicales, se encuentra ampliamente distribuida en el Golfo de México.

Hábitat. En 10-189 m se ha registrado en amplia variedad de sedimentos y asociada a la vegetación; en este estudio se encontró en arena limosa a 16-28 m.

Neanthes micromma Harper, 1979

Referencias. Harper et al. 1979:91, figs. 1-11; Taylor 1984: 31.17, figs. 31.14a-h; Granados Barba 1994: 147

Material examinado. 3 organismos: I(2), 10(1). Distribución. Endémica al Golfo de México. Norte del Golfo de México, Tabasco, Campeche, Veracruz; ahora se cita para Tamaulipas. 
Hábitat. Desde la zona intermareal hasta los $50 \mathrm{~m}$ de profundidad de arena lodosa y arcillosa. En este estudio se encontró en arena limosa a $16-27 \mathrm{~m}$

Nereis grayi Pettibone, 1956

Referencias. Day 1973: 39; Gardiner 1976: 151 , fig. 15 l-n; Pettibone 1956: 282, fig. 3a-g; Taylor 1984: 31-35, figs. 31-33, 34a-f; Miranda Vázquez 1993.

Material examinado. I organismo: 12(1).

Distribución. Atlántico occidental. Nueva Inglaterr?, Carolina del Norte, Veracruz, Yucatán, ésta es la primera cita para Tamaulipas.

Hábitat. Desde la zona intermareal hasta 160 $m$ de profundidad, en fondos arcillosos y finos. En este estudio se encontró en limos a $8 \mathrm{l} \mathrm{m}$

\section{Ceratocephale oculata Banse, 1977}

Referencias. Banse 1977: 620, fig. 3a-f; Gardiner \& Wilson 1979: 165; Taylor 1984:31.9, fig. 6a-e; Granados Barba 1994.

Material examinado. 3 organismos: 1(2), 3(1). Distribución. Anfiamericano. Se ha encontrado ampliamente distribuida en cl Golfo de México y en el Golfo de California.

Hábitat. Se ha registrado en una amplia variedad de sedimentos, desde arena arcillosa hasta fragmentos de conchas y a profundidades de 4,5 a $3.5 \mathrm{~m}$. En este trabajo se encontró en arena limosa a $28 \mathrm{~m}$.

FAMULIA GLYCERIDAE Grube, 1857 Glycera papillosa Grube, 1857

Referencias. Day 1967: 358, fig. 16.1, j-i; 1973: 45; Gilbert 1984: 32-10, figs. 32.5, 6a-e; Rodríguez Villanueva 1993.

Material examinado. I organismo: $8(1)$.

Distribución. Cosmopolita. Sudáfrica, Carolina del Norte, norte del Golfo de México, Texas, Florida, Perú, Chile, en aguas nacionales se ha registrado para Campeche, Tabasco y es primer registro para Tamaulipas.

Hábitat. Desde la zona intermareal hasta 200 $m$ de prolundidad, se ha encontrado en arenas finas y gruesas. En este estudio se registró en arena a 52 :n.
Glycera sphyrabrancha Schmarda, 1861

Referencias. Day, 1973: 47, figs. 6d-g; Gardiner, 1976: 162, fig. 18b-g; Gilbert, 1984:3213, figs. 32-7, 8a-g.

Material examinado. 2 organismos: $1(1), 4(1)$.

Distribución. Atlántico occidental. Carolina del Norte, Florida, norte del Golfo de México hasta las Antillas menores, éste es el primer registro para aguas mexicanas.

Hábitat. En fondos de arena media a gruesa en 15-20 m; en este trabajo se encontró en limos y arena limosa a $18-27 \mathrm{~m}$.

FAMILIA GONIADIDAE Kinberg, 1866

Goniada teres Treadwell, 1931

Referencias. Day, 1973: 51, fig. 7 1-n; Gilbert, 1984: 33-7, fig. 33-3, 4a-n; Rodríguez Villanueva 1993.

Material examinado. 4 organismos: $8(1), 9(1)$, 10(1), 11(1).

Distribución. Atlántico occidental . Carolina del Norte hasta las Antillas menores, ampliamente distribuido en el norte del Golfo de México, Veracruz, Yucatán y es primer registro para Tamaulipas.

Hábitat. Desde aguas someras hasta $200 \mathrm{~m}$ de profundidad. Es una amplia variedad de sedimentos. En este trabajo se registró en arena limosa, limo arenoso y arenas a profundidades de 16 hasta $52 \mathrm{~m}$.

Goniada littorea Hartman, 1950

Referencias. Hartman, 1950: 23, pl. 3, figs. 1-10; Gilbert, 1984: 33.7; Rodríguez Villanueva 1993.

Material examinado. 5 organismos: 8(4), 10(1) Distribución. Anfiamericano. Carolina del Norte, ampliamente distribuida en el norte del Golfo de México, sureste de Califomia, Baja Califomia Norte, Veracruz; éste es el primer registro para Tamaulipas.

Hábitat. En aguas someras de 15 a $65 \mathrm{~m}$ de profundidad en fondo de arena arcillosa a arena media; se encontró en arena limosa y arena a 16-52 m. 
FAMILIA NEPHTYIDAE Grube 1850 Aglaophamus verrilli (McIntosh, 1885)

Referencias. Pettibone, 1963: 190, fig. 48c,d; Day, 1973: 42; Gardiner, 1976: 155, figs. 16 k, 17 a,b; Taylor, 1984: 35-15, figs. 35.11, 12a-c; Granados Barba 1994: 163, Lám. 25b, f, h.

Material examinado. 10 organismos: 15(5), 1.1(4), 12(1).

Distribución. Anfiamericano. Se ha registrado en aguas tropicales, subtropicales y templadas del Atlántico occidental, Golfo de California y se encuentra ampliamente distribuida en el Golfo de México.

Hábitat. Desde aguas someras a $4 \mathrm{~m}$ hasta 189 m de profundidad; estuarina, se ha encontrado en una amplia variedad de sedimento desde lodos hasta fragmentos de conchas, se ha registrado en este trabajo en limos hasta arenas, en $16-81 \mathrm{~m}$.

Aglaophamus circinata (Verrill, 1874)

Referencias. Pettibone, 1963: 192, fig. 48a; Gardiner, 1976: 157, fig. 17c; Taylor, 1984: 35.17. figs. 35. 14a, b; Granados Barba 1994: 163, Lám. 25b, c, h.

Material examinado. 1 organismo: 10(1).

Distribución. Atlántico occidental. Se ha encontrado en aguas templadas y subtropicales del noroeste del Atlántico; registrándose en el Golfo de San Lorenzo, Nueva Escocia, Massachusetts, y norte del Golfo de México, Campeche y Tamaulipas

Hábitat. Se ha encontrado en amplia variedad de sedimentos desde lodos hasta rocas con profundidades desde los 10 hasta los $787 \mathrm{~m}$. En este estudio se encontró en arena limosa a $16 \mathrm{~m}$.

Nephtys incisa Malmgren, 1865

Referencias. Pettibone, 1963: 198, figs. 49a,b, 5 I a; Day, 1973: 43; Taylor, 1984: 35-7, figs. 353, 4a-d; Granados Barba 1994: 163, Lám. 25a, b,e.

Material examinado. 3 organismos: $2(1), 9(1)$, 15(1).

Distribución. Cosmopolita. En Groenlandia, Islandia, Noruega del mar Báltico a Portugal, Gol- fo de San Lorenzo, Nueva Escocia, Mar Mediterráneo; Atlántico norte, en ambas costas de Norteamérica, ampliamente distribuido en el Golfo de México. Es cuestionable que sea la misma entidad biológica, por su amplia distribución; todos los registros requieren confirmación y se sugiere que se revise el material tipo de esta especie para las diferentes áreas geográficas.

Hábitat. Estuarina, costera de aguas someras hasta $1800 \mathrm{~m}$ de profundidad y se ha encontrado en una amplia variedad de sedimentos, desde limos hasta grava; en este estudio se encontró en limos, arena limosa y arenas a 23-60 m.

FAMILIA ONUPHIDAE Kinberg, 1865 Mooreonuphis pallidula (Hartman, 1965)

Referencias. Fauchald, 1982: 62, fig. 17b; Gathof, 1984: 39-25, fig. 39-21, 22a-i

Material examinado. 3 organismos: $8(1), 10(1)$, 13(1).

Distribución. Atlántico occidental, desde Nueva Inglaterra hasta el norte de Brasil; en el norte del Golfo de México se ha registrado en Florida, Alabama y Texas; ahora se cita para aguas mexicanas del Golfo de México.

Hábitat. En fondos de arena fina a gruesa a profundidades de 200 hasta $805 \mathrm{~m}$. En este estudio se encontraron en arena limosa y arenas a 16-52 m.

Onuphis eremita oculata Hartman, 1951

Referencias. Hartman, 1951: 52, pl. 14, figs.I,2; Gathof, 1984: 39-27, figs. 39-25, 26a-j; Hernández-Alcántara 1992: 255.

Material examinado. 2 organismos: 5(1), 10(1). Distribución. Anfiamericano. Se ha registrado en Sinaloa, se encuentra ampliamente distribuido en el norte de Golfo de México hasta Tampico, Tamaulipas.

Hábitat. Desde la zona intermareal hasta 175 $m$ de profundidad, en arcillas hasta arena media. En este estudio se encontró en arena limosa $y$ arena, a $16 \mathrm{~m}$.

FAMILIA LL'MBRINERIDAE Malmgren, 1867

Lumbrinerides acuta (Verrill, 1875) 
Referencias. Gardiner, 1976: 203, fig. 26i-l; Perkins, 1979: 419, fig. Ia; Uebelacker \& Johnson (1984a), 1984b: 41:11, figs. 41.8a-g; Solís-Weiss et al. 1995: 65.

Material examinado. I organismo: $8(1)$.

Distribución. Cosmopolita. Se ha registradoen el sur de África, Nueva York, Nueva Inglaterra, Bermudas, Baja California Sur, norte del Golfo de México, Yucatán, y ésta es la primera cita para Tamaulipas.

Hábitat. desde 12 hasta los $189 \mathrm{~m}$ de profundi$\mathrm{dad}$, en arena fina a grava. En este trabajo se encontró en arena a $52 \mathrm{~m}$.

Lumbrineriopsis paradoxa (Saint Joseph, 1888)

Referencias. Fauvel, 1923: 434, fig. 173 a,h; Gardiner, 1976: 205, figs. 26m-o; Uebelacker \& Johnson (1984a), 1984b: 41.15, fig. 41.12a-f; Solís-Weiss et al. 1995: 66

Material examinado. I organismo: $9(1)$.

Distribución. Trasatlántico. Se ha registrado en las azores en el oeste de África, Carolina del Norte, Bermudas y norte del Golfo de México, Tabasco, Campeche, Yucatán, ahora se cita para Tamaulipas.

Hábitat. Se ha encontrado en una amplia variedad de sedimentos desde limos hasta gravas, en 44-1700 m; en este trabajo se encontró en arena a $34 \mathrm{~m}$.

Scoletoma verrilli Perkins, 1979

Referencias. Perkins, 1979: 44 I, figs. 10, 11 ; Uebelacker \& Johnson (1984a), 1984: 41.24, figs. 41.20a-h; Frame, 1992: 203; Solis-Weisset al. 1995: 71.

Material examinado. 6 organismos: 4(1),8(1), $9(1), 10(3)$

Distribución. Atlántico occidental. Su encuentra ampliamente distribuido en el Golfo de México, Florida y Cuba.

Hábitat. Se ha registrado en sedimento lodoso, arena gruesa y en coral muerto a profundidades de 3 hasta $189 \mathrm{~m}$; en este trabajo se encontró en limos, arena limosa y arena a 16-52 m.

Lumbrineris coccinea (Renier, 1804)

Referencias. Fauvel, 1923: 432, fig. 172g-n;
Day, 1967: 436, fig. 17.16i-m; 1973: 59; Uebelacker \& Johnson (1984a), 1984b:41-35, figs. 4 J-31,32 a-i; Solís-Weiss et al. 1995: 67.

Material examinado. I organismo: I1(1).

Distribución. Cosmopolita. Se ha registrado en Alaska, Antillas, África, Inglaterra, Mar Mediterráneo, Japón, Golfo de California, se encuentrà ampliamente distribuido en el norte del Golfo de México, Veracruz, Campeche, Yucatán, Eermudas y Cuba. Es cuestionable que sea la misma entidad biológica por su amplia distribución; todos los registros recuieren confirmación y se sugiere que se revise el material tipo de esta especie para las difcrentes áreas geográficas.

Hábitat. Desde la zona intermareal hasta los $1300 \mathrm{~m}$ y se ha encontrado en una amplia variedad de sedimento desde arena fina a gruesa y es muy común en corales, rocas y asociados en algas. Para este trabajo se registró en limo arenoso a $33 \mathrm{~m}$.

Lumbrineris inflata Moore, 1911

Referencias. Uay, 1967: 435, figs. 17.16d-h; 1973: 59; Gardiner, 1976: 198, figs. 25n-q; Uebelacker \& Johnson (1984a), 1.984b: 41.37, figs. 41.34a-g; Miranda Vázquez 1993

Material examinado. I organismo: $8(1)$ Distribución. Circuntropical, ampliamente distribuida en el noreste del Golfo de México, Baja California, Baja California Sur, Campeche, Yucatán, Tabasco y Tamaulipas.

Hábitat. Intermarcal hasta $130 \mathrm{~m}$; en fondos de lodo, grava con fragmentos de conchas, rocas, coral. En este estudio se recolectó en arena a $52 \mathrm{~m}$.

FAMILIA DORVILLEIDAE Chamberlin, 1919

Schistomeringos pectinala Perkin.s, 1979

Referencias. Perkins, 1979: 456, figs. 18-20; Wolf, 1984: 44-20, fig:: 44-13, 14a-t; Rodríguez Villan icva 1993.

Material examinado. 2 organismos: 7(2). Distribución. Endémica al Golfo de México. Se ha registrado para Florida, norte del Golfo de México, primera cita para Tamaulipas.

Hábitat. Se ha encuntrado en arena fina a gruesa y arcilla a $7-11 \mathrm{~m}$; Para este trabajo se encontró en sedimento limo arenoso a $32 \mathrm{~m}$. 


\section{DISCUSIÓN}

En el litoral de Tamilulipas sólo se habían registrado 64 especies de noliquetos, y con este estudio el númcro de especies se incrementa a 79 , ya que de las 24 especies que se presentan en este trabajo, 1 ; son nuevos registros para la costa occidental del Golfo de México; estos registros indican la falta de estudios de poliquetos y demuestran la necesidad de realizar trabajos que sigan incrementado su conocimiento en nuestras costas.

De lats 24 especies identificadas para Tamaulipas la mayoría ha sido previamente observada en el Golfo de México y el Atlántico, debido a la influencia directa de aguas tropicales y subtropicales del Golfo de México. Sin embargo, aunque la fauna poliquetológica es muy similar existen diferencias en cuanto a su distribución y abundancia, debido al comportamiento ontogénico de cada una de las especies.

Las especies Anfiamericanas son aquilas que se presentan en ambas costas de América y su elevado porcentaje se explica a que hace 3 a 3,5 millones de años hubo un intercambio de fauna a través del estrecho de Panamá cuando este aún no se formaba (Brigss, 1974, SalazarVallejo, 1986).

Los cambios físicos que se presentaron a causa del cierre del estrecho panámico causaron la extinción de algunas uspecies, la sobrevivencia de otras y evolución de nuevas formas (Laguna, 1990); esto probablemente sucedió con las especies restringidas al Golfo de México (endémicas) y debido a que no tienen una distribución tan amplia como las del Atlántico occidental o cosmopolita éstas exhiben mayores rangos de distribución por ser eurioicas y pueden resistir a las diferentes rangos de temperaturas que se presenten en las diferentes latitudes.

\section{AGRADECIMIENTOS}

El presente trabajo forma parte de un proyecto institucional denominado Estudio de las Condiciones Oceanográficas del Litoral de Tamaulipas, sufragado por la Estación de Investigación Oceanográfica de Tampico de la Dirección General de Oceanografía Naval. La Universidad de Quintana Roo me proporcionó tiempo y espacio para concluir el manuscrito. Agradezco a Sergio I. Salazar-Vallejo, los comentarios y sugerencias al escrito ya que mejoró en gran parte; Juan Manuel Salazar Felipe, elaboró la figura; Michael Cunniffe McEvoy mejoró el resumen en inglés.

\section{LITERATURA CITADA}

Banse, K. 1977. Gymnonereidinae new subfamily: the Nereididae (Polychaeta) with bifid parapodial neurocirri. J. Nat. Hist. 11: 609-628.

Brigss, J. C. 1974. Marine Zoogeography. McGraw-Hill, xi, 475 pp.

Day, J. H. 1967. A monograph on the polychaeta of Southern África. Brit. Mus. (Nat. Hist.), Publ. 656: 38-878.

Day, J. H. 1973. New Polychaeta from Beaufort, with a key to all species recorded from North Carolina. NOAA Tech. Rep. NMFS 75: 8-140.

De León González, J.A. 1985. Eunicidae (Polychaeta) de 10 localidades de las costas mexicanas. Tes. Prof., Fac. CC. BB., UANL, $53 \mathrm{pp}$.

Dexter, M. D. 1976. The Sandy-beach fauna of México. Southw. Nat. 20(4): $479-$ $485 \mathrm{pp}$.

Fauchald, K. 1972. Benthic polychaetous annelids from deep water of Western Mexico and adjacent areas in the Eastern Pacific Ocean. Allan Hanckock Monogr. Mar. Biol., 7 pp. 
Fauchald, K. 1977. The Polychaete worms. Definitions and Keys to the orders, families and genera. Nat. Mus. Hist. L. A. Sci. Ser. 28: 1-190.

Fauchald, K. \& P. A. Jumars. 1979. The diet of worms: A study of polychaete feeding guide. Oceanogr. Mar. Biol. Ann. Rev. 17: 193-284.

Fauchald, K. 1982. Two new species of Onuphis (Onuphidae: Polychaeta) from Uruguay. Proc. Biol. Soc. Wash. 93(1): 203-209.

Fauvel, P. 1923. Polychètes errantes. Faune de France, 16: 1-494.

Frame, A. B. 1992. The Lumbrinerids (Annelida: Polychaeta) collected in two Northwestern Atlantic surveys with descriptions of a new genus and two new species. Proc. Biol. Soc. Wash., 105(2): 185-218.

Gardiner, S. L. 1976. Errant polychaete annelids from North Carolina. J. Elisha Mitchell Sci. Soc. 91: 77-270

Gardiner, S. L. \& W. H. Wilson, Jr. 1979. New records of polychaete annelids from North Carolina with the description of a new species of Sphaerosyllis (Syllidae). J Elisha Mitchell Sci. Soc. 93(4): 9-172.

Gathof, J. M. 1984. Phyllodocidae, Onuphidae. In Uebelacker, J. M. \& P. G. Johnson (eds.). Taxonomic guide to the polychaetes of the Northern Gulf of Mexico. Final Report to the Minerals Management Service, contract 14-12001-29091. Barry A. Vittor \& Assoc., Inc., Mobile, Alabama. 7 Vols.: 19.6, $19.23,39.25 ; 39-27 . \mathrm{pp}$

Gilbert, K. M. 1984. Glyceridae; Goniadidae. In Uebelacker, J. M. \& P. G. Johnson (eds.) Taxonomic guide to the polychaetes of the northern Gulf of Mexico. Final Report to the Minerals Management Service, contract 14-12-001-29091. Barry A.
Vittor \& Assoc., Inc., Mobile, Alabama. 7 Vols.: $32.10,32.13,33.7$

Granados Barba, A. 1994. Estudio sistemático de los poliquetos (Annelida: Polychaeta) de la región de plataformas petroleras del sur del Golfo de México. Tesis de Maestría, Univ. Autónoma de México, 247 pp.

Harper, D. E., C. R. Shelton, G.E. Williams \& K.W. Johnson. 1979. The Ocurrence of Pisionides indica (Polychaeta: Pisionidae) in the Western Gulf of México. Tex. J. Sci., 31 (1): 39-41.

Hartman, O. 1950. Goniadidae, Glyceridae and Nephtyidae. Allan Hancock Found. Pac. Exped. 15(1): 1-181.

Hartman, O. 1951. The littoral marine annelids of the Gulf of México. Publ. Univ. Tex. Inst. Mar. Sci., 2: 7-124.

Hartman, O. 1963. Submarine canyons of Southern Califomia. Part III. Systematics: Polychaetes. Allan Hancock Found. Pacific. Exped. 27: 1-93.

Hartman, O. 1968. Atlas of Frrantiate Polychaetous Annelids from Califomia. Allan Hancock Found., Univ. Sou. Cal., $828 \mathrm{pp}$.

Hernández-Alcántara, P. 1992. Los poliquetos (Annelida: Polychaeta) de la plataforma continental del Golfo de California, México. Taxonomía, abundancia numérica y distribución geográfica. Tesis de Maestría, Universidad Nacional Autónoma de México, 427 pp.

Hemández-Alcantara, P. Y V. Solís-Weiss. 1993 Distribución latitudinal y batimétrica de los anélidos poliquetos del orden TEREBELLOMORPHA de la plataforma continental del Golfo de California, México. Cuad. Mex. Zool. I(2): 65-72.

Laguna, J.E. 1990. Shore barnacles (Cirripedia, Thoracica) and a revision of their 
provincialism and transition zones in the Tropical eastern Pacific Bull. Mar. Sci., 46(2): 406-424.

Lugo, H. 1985. Morfoestructuras del fondo oceánico mexicano. Bol. Inst. Geogr. UNAM, (15): $293 \mathrm{pp}$.

Méndez Ubach, M. N. 1983. Relaciones entre fauna y sedimento en 29 playas arenosas del Golfo de México. Tes. Prof., Fac. Cienc., UNAM, 103 pp.

Miranda Vázquez, L. A. 1993. Estudio de las comunidades de poliquetos (Annelida: Polychaeta) de la plataforma continental externa del sur del Golfo de México. Tesis Profesional Facultad Ciencias, UNAM, $148 \mathrm{pp}$

Pettibone, M. H. 1956. Marine polychaete worms from Labrador. Proc. U. S. Nat. Mus. 105: 531-584.

Pettibone, M. H. 1963. Marine polychaete worms of the New England region. Aphroditidae through Trochochaetidae Bull. U. S. Natl. Mus. 227: I-356.

Pettibone, M. H. 1966. Revision of Pilargidae (Annelida: Polychaeta) including descriptions of new species and redescription of the pelagic Podarmaus ploa Chamberlin (Polynoidae). Proc. U. S. Natl. Mus. 118(3525): 155-207.

Perkins, T. H. 1979. Lumbrineridae, Arabellidae, and Dorvilleidae (Polychaeta), principally from Florida with descriptions of six new species. Proc. Biol. Soc. Wash. 92: 415 465.

Perkins, T. H. 1981. Syllidae (Polychaeta), principally from Florida, with descriptions of a new genus and twenty-one new species. Proc. Biol. Soc. Wash. 93(4): 1080-1172.
Pica Granados, Y., M. G. Ponce Vélez \& M. E. Barrón Echaury. 1991. Oceanografía geológica del Golfo de México y Mar Caribe Mexicano En: De La Lanza Espino (Comp.). Oceanografía de Mares Mexicanos. AGT Editor, México D.F.

Rodríguez Villanueva, L. V. 1993. Los poliquetos (Annelida: Polychaeta) de la plataforma continental interna del sur del Golfo de México; abundancia, distribución y diversidad. Tesis Profesional, Facultad Ciencias, UNAM, $128 \mathrm{pp}$.

Salazar-Vallejo, S. I. 1981. La colección de poliquetos (Annelida: Polychaeta) de la Facultad de Ciencias Biológicas de la Universidad Autónoma de Nuevo León, México. Tes. Prof. Fac. Cienc. Biol. Univ. Autón. N. León, 156 pp.

Salazar-Vallejo, S. I. 1986. Pilargidea (Annelida: Polychaeta) de México: Lista de especie, nueva especie y biografía, Cahiers De Biologie Marine 28: 193-209.

Salazar-Vallejo, S. I., J. A. De León-González \& H. Salaices- Polanco. 1989 Poliquetos (Annelida: Polychaeta) de México. Generalidades, claves ilustradas para familias y géneros, bibliografía y lista de especies. Libros Univ. Autón. Baja Calif. Sur, La Paz, 211 pp.

Salazar-Vallejo, S. I. 1996. Filodócidos (Polychaeta: Phyllodocidae) del Caribe Mexicano con claves para identificar las especies del Gran Caribe. Rev. Biol. Trop., 44(1): 107-122.

Solís-Weiss. V., Rodríguez Villanueva, L. V., Granados Barba, A. Ochoa Rivera, V., Miranda Vázquez, L. \& P. Hernández Alcántara. 1995. The Lumbrineridae of the continental shelf in the Mexican portion of the Gulf of Mexico. Mitt. Hamb. Zool. Mus. Inst. 92(suppl.): 61-75. 
Taylor, J. L. 1984. Nereididae; Nephtyidae. In Uebelacker, J. M. \& P. G. Johnson, (eds.). Taxonomic guide to the polychaetes of the Northern Gulf of México. Final Report to the Minerals Management Service, contract 14-12001-29091. Barry A. Vittor \& Assoc., Inc., Mobile, Alabama. 7 Vols.: 31.9, $31.17,35.15$.

Uebelacker, J. M. \& P. G. Johnson (eds.). $1984 a$. Taxonomic Guide to the Polychaetes of the Northem Gulf of Mexico. Final Report to the Minerals Management Service, contrac 14-12-001-29091. Barry A. Vittor \& Assoc., Inc., Mobile, Alabama. 7 Vols.

Uebelacker, J. M. \& P. G. Johnson. 1984b. Syllidae, Lumbrineridae. In Uebelacker, J. M. \& P. G. Johnson (eds). Taxonomic Guide to the Polychaetes of the Northern
Gulf of Mexico. Final Report to the Minerals Management Service, contract 14-12-001-29091. Barry A. Vittor \& Assoc., Inc., Mobile, Alabama. 7 Vols.: $30.29,30.33,41.11,41.15,41.24,41.35$.

Vidal Lorandi, F. V., A. F. L. Vidal, E. O. Hernández, M. Conde y L. S. Zambrano. (1994). Atlas Oceanográfico del Golfo de México. Vol. III. Ed. Inst. Inv. Eléc. $579 \mathrm{pp}$

Wolf, P. S. 1984. Pilargidae, Dorvilleidae. In Uebelacker, J. M. \& P. G. Johnson (eds.). Taxonomic guide to the polychaetes of the Northern Gulf of México. Final Report to the Minerals Management Service, contract 14-12001-2909l. Barry A. Vittor \& Assoc., Inc., Mobile, Alabama. 7 Vols. 